\title{
Level Density and Strength Function from Quasicontinuous Decay of Superdeformed Excitations in Lead Nuclei
}

Jolie A. Cizewski, Micah Johnson, and Dennis P. McNabb

October 4, 2002

U.S. Department of Energy

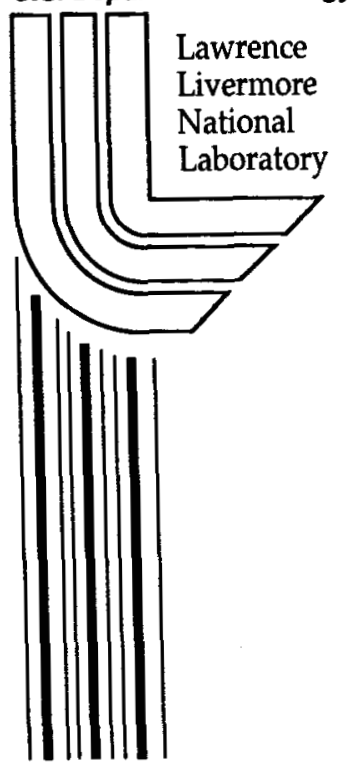




\section{DISCLAIMER}

This document was prepared as an account of work sponsored by an agency of the United States Government. Neither the United States Government nor the University of California nor any of their employees, makes any warranty, express or implied, or assumes any legal liability or responsibility for the accuracy, completeness, or usefulness of any information, apparatus, product, or process disclosed, or represents that its use would not infringe privately owned rights. Reference herein to any specific commercial product, process, or service by trade name, trademark, manufacturer, or otherwise, does not necessarily constitute or imply its endorsement, recommendation, or favoring by the United States Government or the University of California. The views and opinions of authors expressed herein do not necessarily state or reflect those of the United States Government or the University of California, and shall not be used for advertising or product endorsement purposes.

This work was performed under the auspices of the U. S. Department of Energy by the University of California, Lawrence Livermore National Laboratory under Contract No. W-7405-Eng-48.

This report has been reproduced directly from the best available copy.

Available electronically at http://www.doc.gov/bridge

Available for a processing fee to U.S. Department of Energy

And its contractors in paper from

U.S. Department of Energy

Office of Scientific and Technical Information

P.O. Box 62

Oak Ridge, TN 37831-0062

Telephone: (865) 576-8401

Facsimile: (865) 576-5728

E-mail: reports@adonis.osti.gov

Available for the sale to the public from

U.S. Department of Commerce

National Technical Information Service

5285 Port Royal Road

Springfield, VA 22161

Telephone: (800) 553-6847

Facsimile: (703) 605-6900

E-mail: orders@ntis.fedworld.gov

Online ordering: http://www.ntis.gov/ordering.htm

\section{OR}

Lawrence Livermore National Laboratory

Technical Information Department's Digital Library

http://www.llnl.gov/tid/Library.html 


\title{
LEVEL DENSITY AND STRENGTH FUNCTION FROM QUASICONTINUOUS DECAY OF SUPERDEFORMED EXCITATIONS IN LEAD NUCLEI
}

\author{
JOLIE A. CIZEWSKI, MICAH JOHNSON \\ Department of Physics and Astronomy, Rutgers University, New Brunswick, NJ \\ 08854-8019 USA \\ DENNIS P. MCNABB \\ Nuclear Science Divison, Lawrence Livermore National Laboratory, Livermore, \\ CA 94550 USA
}

The shape of the spectrum associated with the quasicontinuous (QC) decay of superdeformed rotational bands in even- and odd-mass $\mathrm{Pb}$ isotopes is sensitive to the gap in level density at finite temperature and angular momentum at normal deformations. This gap in level density was deduced to be $\sim 0.95 \mathrm{MeV}$ at $6 \hbar$ for ${ }^{194} \mathrm{~Pb}$ and $\sim 0.4 \mathrm{MeV}$ at $10 \hbar$ for ${ }^{192} \mathrm{~Pb}$, while the shape of the $\mathrm{QC}$ spectrum for ${ }^{195} \mathrm{~Pb}$ is consistent with no gap in the level density at about $11 \hbar$.

\section{Introduction}

Nuclear excitations at large elongations, superdeformed (SD) shapes, have been observed in many nuclei in the $A \sim 190$ region. These excitations at high angular momenta are identified by the characteristic $\boldsymbol{\gamma}$-ray spectrum of equally-spaced transitions, where the spacing is inversely proportional to the moment of inertia. A particular example is ${ }^{194} \mathrm{~Pb}$ where the superdeformed rotational band has been observed down to the $6^{+}$state and 12 one-step transitions between SD and excitations with normal deformations (ND) have been identified ${ }^{1,2}$. However, these one-step transitions only account for about $20 \%$ of the intensity of the SD band. In other nuclei in the $A \sim 190$ region one-step transitions account for at most $5 \%$ of the total SD intentisy. The present paper will highlight the dominant mode by which SD bands decay: multistep processes between the SD and ND states which form a quasicontinuous (QC) spectrum. 


\section{Experimental Procedures}

These measurements have been a collaboration of scientists from Argonne, Livermore and Berkeley National Laboratories, the Institut de Recherches at Strasbourg, and Rutgers University. The experiments were performed at the Lawrence Berkeley National Laboratory 88-Inch Cyclotron facility and $\gamma$-ray spectroscopy was enabled by Gammasphere with $88-95$ Comptonsuppressed Ge detectors. The reactions involved ${ }^{24,25,26} \mathrm{Mg}$ beams on goldbacked ${ }^{173,174} \mathrm{Yb}$ targets to populate SD excitations in ${ }^{192,194,195} \mathrm{~Pb}$. The response function of Gammasphere had previously been determined and used to unfold the $\gamma$-ray spectra, correct for efficiency, and account for summing events, using standard techniques ${ }^{3,4}$.

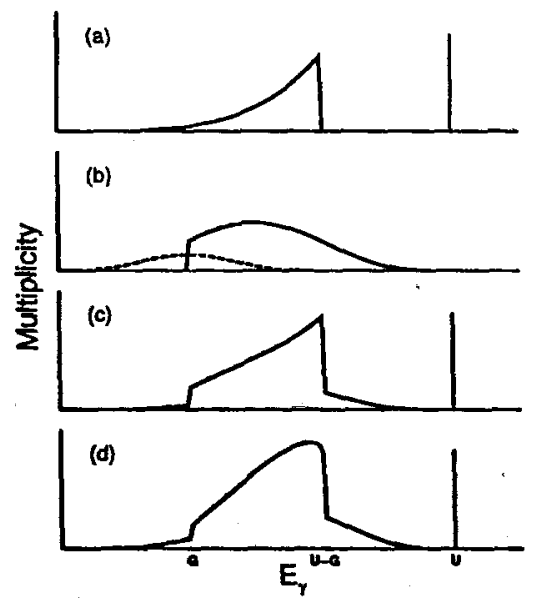

Figure 1. Schematic model of the QC decay of SD bands in even-even nuclei. (a) First step; (b) Second step (dotted transitions terminate above the ND line); (c) QC spectrum for 2-step decay; (d) QC spectrum for 2-step decay with back-shifted Fermi gas.

\section{Population and Decay of Superdeformed Excitations}

The residual superdeformed nucleus is populated at high energy and angular momentum following evaporation of neutrons from a hot residue following the heavy-ion fusion reaction. The initial de-excitation is via statistical $\gamma$ rays, predominantly of $\mathrm{E} 1$ character, which cool the evaporation residue to excitations within $\sim 1 \mathrm{MeV}$ of the yrast SD line, bound within the second well of the potential energy surface. The nucleus continues to lose energy 
and angular momentum via mixed M1/E2 transitions between excited SD bands, and stretched $\mathrm{E} 2$ transitions between excited SD bands, until the yrast SD line is reached, and the decay proceeds via the characteristic equally spaced enhanced E2 $\gamma$-ray transitions which were used to identify the $\mathrm{SD}$ excitations. At some point the nucleus is several $\mathrm{MeV}$ in excitation above the normal yrast line. Although the transitions within the SD band are highly collective, these states at the bottom of the SD band mix with levels with similar quantum numbers at normal deformation and the energy factor enhances the transition probabilities to states of normal deformation, towards the ND yrast line.

Since the decay of SD excitations proceeds via mixing with states with normal deformations at the same energy and angular momentum, the quasicontinuous spectrum associated with the decay of these SD bands represents the decay of excitations at finite energy, $2-4 \mathrm{MeV}$ above the yrast line, and finite angular momentum, 6-12 .

The expected shape of this QC decay spectrum can be predicted by a simple schematic model ${ }^{5}$ in which the level density is approximated by constant level spacing up to the excitation energy, U, above the yrast line, given by the energy above yrast at which the SD band decays to ND excitations. As a result of pairing correlations, a gap of energy $G$ between the yrast line at a given spin and the onset of this simple level density is expected in even-even nuclei. The $\gamma$-ray strength function can be approximated by an exponential dependence on $\gamma$-ray energy, where $E_{\gamma}^{4}$ can be chosen to approximate the effects of the tail of the giant dipole resonance for E1 transitions. The $\gamma$-ray spectrum associated with the first step of the decay in this schematic model is shown in Fig. 1a: a discrete peak at energy $\mathrm{U}$, for the transition to the ND yrast line, with the next highest transition at energy U-G, for the highest energy transition to states above the yrast line, followed by an exponential decrease in intensity for lower energy transitions. The initial states for the second step of the decay are the final states from the first step of the decay. The spectrum associated with the second step in the SD decay has two components. The first is for transitions which terminate at the yrast line. The highest transition is of energy $U$, with very low intensity, and lowest energy transition with energy G. The other component of the second step in the QC decay is transitions which terminate above the yrast line, and is a spectrum which is a convolution of the QC spectrum in the first step with itself. The unresolved decay components for the SD bands in ${ }^{192,194,195} \mathrm{~Pb}$ are observed to be dominated by two-step pathways. Therefore, only 2 steps were included in the schematic 
model, giving rise to the spectrum displayed in Fig. 1c: a discrete peak at energy $U$, with very low intensity for energies down to $U-G$, at which point the intensity dramatically rises. These properties of the QC spectrum are also expected from the more realistic level density Fermi gas model, with a backshift parameter given by $\mathrm{G}$, as shown in Fig. 2d.

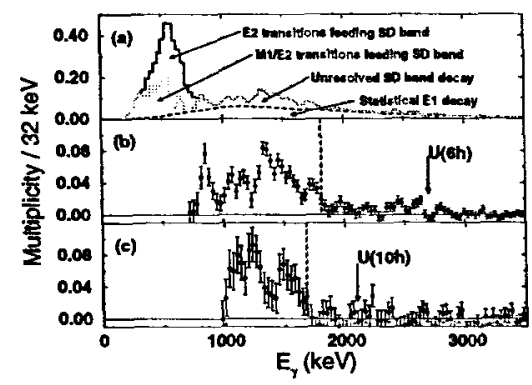

Figure 2. (a) ${ }^{194} \mathrm{~Pb}$ SD-gated spectrum of unresolved transitions. Unresolved transitions that depopulate the SD bands in (b) ${ }^{194} \mathrm{~Pb}$ and (c) ${ }^{192} \mathrm{~Pb}$. Excitation energies, $\mathrm{U}$, of the SD bands relative to the yrast state are indicated.

\section{Quasicontinuous decay spectra of SD bands in even-even $\mathrm{Pb}$ isotopes}

The quasicontinuous components of the SD spectrum associated with the $\mathrm{SD}$ band in ${ }^{194} \mathrm{~Pb}$ are displayed in Fig. 2a. At highest energies are the statistical transitions, which cool the residue after evaporation of neutrons. The stretched E2 transitions between excited SD bands are identified by characteristic angular distributions, which enables a separation from the $\mathrm{QC}$ decay transitions which have more isotropic angular distributions. At lowest energy are the mixed M1/E2 transitions between excited SD bands, again identified by the angular distributions. The transitions associated with the QC decay can then be isolated, and are displayed in Fig. 2b,c.

The spectrum in Fig. $2 \mathrm{~b}$ associated with the QC decay of the SD band in ${ }^{194} \mathrm{~Pb}$ has the shape expected from the schematic model. $U$ is the energy of the one-step discrete transitions, determined previously ${ }^{1,2}$. There is a dramatic rise in intensity at an energy of about $\sim 0.9 \mathrm{MeV}$ below that of the one-step transitions. This dramatic rise is exactly what is expected from a level density formulation with a backshift parameter of $\sim 0.9 \mathrm{MeV}$. In ${ }^{194} \mathrm{~Pb}$ the SD band decays at about $6 \hbar$.

For ${ }^{192} \mathrm{~Pb}$, displayed in Fig. 2c, a one-step transition of $2.0 \mathrm{MeV}$ has 
been observed and the SD band decays to ND excitations at $10 \pi^{6}$ For ${ }^{192} \mathrm{~Pb}$ the gap is deduced to be $\sim 0.4 \mathrm{MeV}$. Therefore, a rapid decrease in gap energy is observed in the $\mathrm{Pb}$ isotopes from the $1.8 \mathrm{MeV}$ at zero spin to about $0.4 \mathrm{MeV}$ at $10 \hbar$. These results suggest that it is probably appropriate to assume no gap in level density, or backshift parameter, when analyzing level densities at high $(>10 \hbar)$ spins.

\section{Quasicontinuous decay spectra of SD bands in ${ }^{195} \mathrm{~Pb}$}

In an odd-mass nucleus no gap in level density is expected. A schematic model with constant temperature level density can be used to predict the shape expected for the QC decay of an SD band, now for an odd-mass nucleus. With no gap in level density, the QC spectrum should smoothly extend towards the excitation energy of the SD band above the yrast line. To normalize the predicted shape, the experimental multiplicity of 2 was used. The resultant spectra for the first and second steps, and total spectrum terminating after 2 steps are shown in Fig. 3.
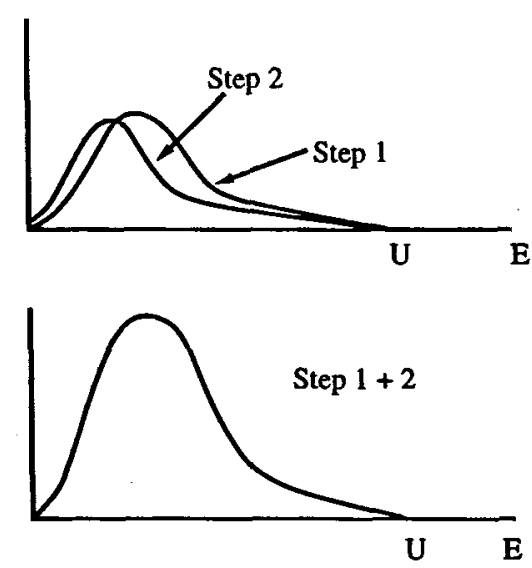

Figure 3. Schematic model of the QC decay of SD bands in an odd-A nucleus. (a) First and second steps of the decay; (b) QC spectrum for 2-step decay.

Four SD bands have been identified in ${ }^{195} \mathrm{~Pb}^{7}$. Since SD bands 1 and 2 are in coincidence with each other at low energy, the sum of the QC spectra associated with these bands are analyzed, and displayed in Fig. 4. The total non-discrete spectrum for ${ }^{195} \mathrm{~Pb}$ has the same components as for even-A isotopes: statistical transitions at high energy which cool the nucleus to excited SD bands which decay by intraband stretched E2 
transitions and interband mixed M1/E2 transitions, and a quasicontinuum of transitions populating the ND states. The statistical transitions at high energy, and the feeding E2 transitions (identified by their characteristic angular distributions) have been subtracted to yield the QC decay spectrum in Fig. 5. This spectrum extends smoothly to high spins - as expected for no gap in level density. In the case of ${ }^{195} \mathrm{~Pb}$ no discrete one-step transitions connecting the SD and ND excitations have been identified. Rather, two methods have been used to estimate the energy above yrast, $U$, of the $\mathrm{SD}$ excitations. In the first the QC decay spectrum is examined at high energy and the value of $U$ is determined as the energy at which the QC spectrum statistically deviates from zero intensity. In the second method, the energy removed by the $\mathrm{QC}$ decay transitions, determined by the average energy of these transitions and the multiplicity of this QC component, is used to determine the energy above yrast. Both methods give a value of $\mathrm{U} \sim 2.6 \mathrm{MeV}$ at $\sim 11 \hbar$, which is similar to the value for ${ }^{194} \mathrm{~Pb}$ determined from the discrete one-step transitions.

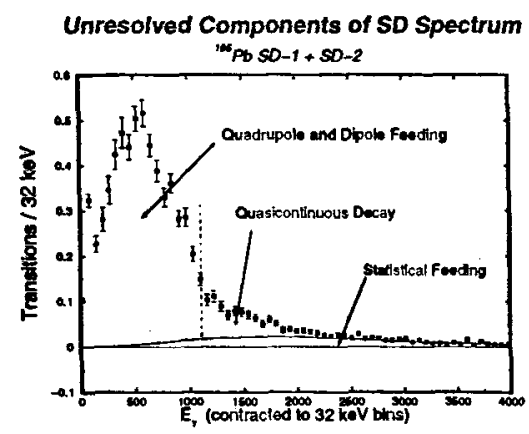

Figure 4. ${ }^{195} \mathrm{~Pb}$ SD-gated spectrum of unresolved transitions.

\section{Summary}

The quasicontinuous decay of superdeformed rotational bands in $192,194,195 \mathrm{~Pb}$ has been used to probe the level density of normal deformed excitations at finite excitation energy and angular momentum. The shape of the QC decay spectra are particularly sensitive to any gap in level density, or backshift parameter. In the even-even ${ }^{192,194} \mathrm{~Pb}$ the QC decay spectra are consistent with a finite gap in level density, which is considerably less than the $1.8 \mathrm{MeV}$ value at zero spin, and decreases from $\sim 0.9 \mathrm{MeV}$ at $6 \hbar$ 


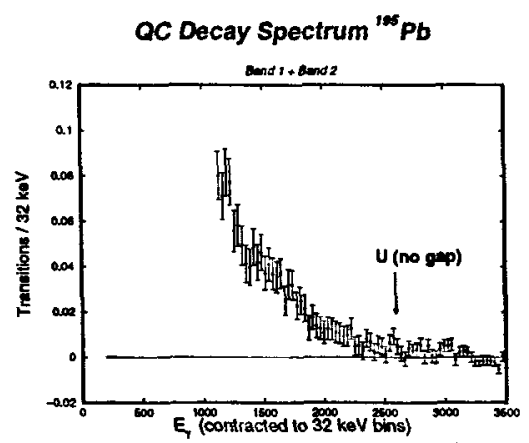

Figure 5. Preliminary results for the $\mathrm{QC}$ decay spectrum for $\mathrm{SD}$ bands $1+2$ in ${ }^{195} \mathrm{~Pb}$.

to $\sim 0.4 \mathrm{MeV}$ at $10 \hbar$. For the odd-mass ${ }^{195} \mathrm{~Pb}$, the spectrum is consistent with no gap in level density, as expected for an odd mass nucleus. From the shape of this QC spectrum an excitation energy above yrast of $\sim 2.6 \mathrm{MeV}$ at $\sim 11 \hbar$ can be extracted.

\section{Acknowledgments}

This work has been supported in part by the U.S. National Science Foundation (Rutgers) and U.S. Department of Energy under Contract No. W7405-ENG-48 (LLNL). We would like to thank our colleagues at Argonne, Livermore, and Berkely National Laboratories for their efforts on the acquisition and interpretation of the experimental results.

\section{References}

1. K Hauschild et al., Phys. Rev. C55, 2819 (1997).

2. A. Lopez-Martens et al., Phys. Rev. Lett. 77, 17071 (1996).

3. D. C. Radford, et al., Nucl. Instrum. Methods Phys. Res. A 258, 111 (1987)

4. R. Holtzmann et al., Phys. Lett. B 195, 321 (1987).

5. D. P. McNabb et al., Phys. Rev. C61, 031304(R) (2000).

6. D. P. McNabb et al., Phys. Rev. C56, 2474 (1997).

7. L. P. Farris et al., Phys. Rev. C51, R2288 (1995). 